\title{
Supercritical accretion disks in ultraluminous X-ray sources and SS 433
}

\author{
Sergei Fabrikaa ${ }^{1,2 \star}$, Yoshihiro Ueda ${ }^{3}$, Alexander Vinokurov ${ }^{1}$, Olga Sholukhova' and Megumi Shidatsu ${ }^{3}$
}

The black hole mass and accretion rate in ultraluminous X-ray sources (ULXs) in external galaxies, whose $X$-ray luminosities exceed those of the brightest black holes in our Galaxy by hundreds and thousands of times ${ }^{1,2}$, is an unsolved problem. Here we report that all ULXs ever spectroscopically observed have almost the same optical spectra, apparently of WNL type (late nitrogen Wolf-Rayet stars) or LBV (luminous blue variables) in their hot state, which are very scarce stellar objects. We show that the spectra do not originate from WNL/LBV-type donors but from very hot winds from the accretion disks with nearly normal hydrogen content, which have similar physical conditions to the stellar winds from these stars. The optical spectra are similar to that of SS 433, the only known supercritical accretor in our Galaxy ${ }^{3}$, although the ULX spectra indicate a higher wind temperature. Our results suggest that ULXs with X-ray luminosities of $\sim 10^{40} \mathrm{erg} \mathrm{s}^{-1}$ must constitute a homogeneous class of objects, which most likely have supercritical accretion disks.

ULXs have luminosities exceeding the Eddington limit for a typical stellar-mass black hole ${ }^{1,2}, \sim 2 \times 10^{39} \mathrm{erg} \mathrm{s}^{-1}$. Despite their importance in understanding the origin of supermassive black holes that reside in most of the present galaxies, several critical questions are still open. What black hole masses do the ULXs contain? Do they possess standard accretion disks? Do they belong to a homogeneous class of objects? The most popular models for the ULXs involve either intermediate mass black holes $\left(\mathrm{IMBH}, 10^{3}-10^{4} M_{\odot}\right)$ with standard accretion disks ${ }^{4}$ or stellar-mass black holes $\left(\sim 10 M_{\odot}\right)$ accreting at super-Eddington rates ${ }^{5}$. Except for transient ULXs, both scenarios require a donor with a high mass-loss rate in a close binary (such as a massive star and an asymptotic giant branch star), which means a short evolution stage.

Although the X-ray properties of the ULXs are astonishing, it has been impossible, from the X-ray data alone, to distinguish even the main ULX models proposed. Optical spectroscopy may provide us with unique information on the ULXs. Because the ULX optical counterparts are very faint targets ( $\sim 22-24$ magnitude), however, very deep observations with the largest telescopes are required.

Using the Japanese $8.2 \mathrm{~m}$ telescope Subaru, we have obtained highest-quality optical spectra of four nearest, bona fide ULXs that have unambiguous optical counterparts, single star-like objects-Holmberg II X-1, Holmberg IX X-1, NGC 4559 X-7 and NGC 5204 X-1. All details on the observations and data reduction can be found in Supplementary Section 1.

Figure 1 shows the spectra of the ULX optical counterparts (the full range data are given in Supplementary Fig. 1). Main features in all the spectra are the bright He II $\lambda 4686$, and hydrogen $\mathrm{H}_{\alpha}$ and $\mathrm{H}_{\beta}$ emission lines. The lines are obviously broad; the widths range from 500 to $1,500 \mathrm{~km} \mathrm{~s}^{-1}$. In some objects we detect
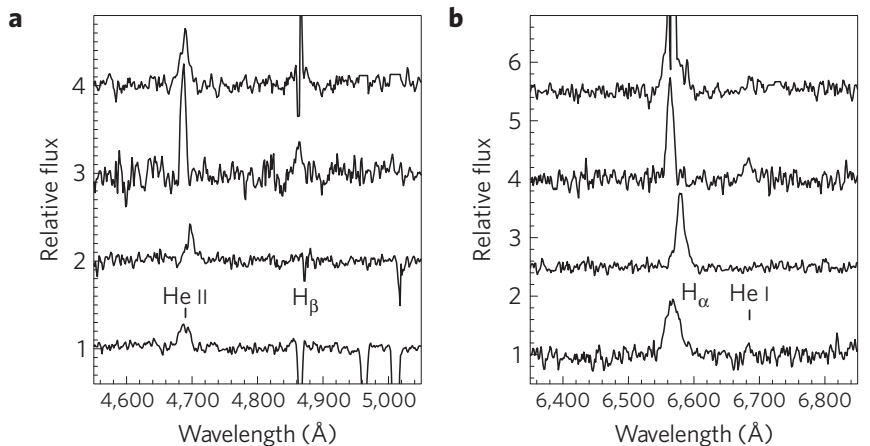

Figure 1 | Spectra of the ULX optical counterparts. Spectra of Holmberg II, Holmberg IX, NGC 4559 and NGC 5204 (from top to bottom) in blue (a) and red (b) spectral regions. The spectra are normalized for better inspection. The strongest are the He II $\lambda 4686$ line and the hydrogen lines $\mathrm{H}_{\alpha} \lambda 6563$ and $\mathrm{H}_{\beta} \lambda 4861$. The broad $\mathrm{He} \mathrm{I} \lambda 6678$ line is also detected. Narrow nebular emission in $\mathrm{H}_{\beta}$ and [O III] $\lambda \lambda 4959,5007$ lines is oversubtracted in the bottom two spectra. Although the hydrogen lines are contaminated with the nebular emission, their broad wings are clearly seen.

broad He I $\lambda 6678,5876$ emission lines. The spectra are very blue (Supplementary Fig. 1), in agreement with the photometric results ${ }^{6}$.

We reveal that the ULX spectra are similar to those of WNL stars, or extreme hot Of supergiants-transition stars, or those of LBVs in their compact hot states ${ }^{7-9}$. All these are massive stars in neighbouring evolutional stages. This indicates the presence of hot outflow in the binary system: it could be a stellar wind from the donor, an irradiated surface of the accretion disk, or a powerful disk wind. The spectra are also similar to that of SS 433 (ref. 10), the only known supercritical accretor in our Galaxy, a close binary consisting of an A-type supergiant and a stellar-mass black hole. SS 433 apparently exhibits a WNL-type spectrum ${ }^{3,11}$ because the physical conditions of its disk wind may be similar to those of stellar winds from WNL stars. The impression of the spectral similarity is strengthened because the absolute visual magnitudes of both WNLs and SS 433 are in the same range as in the ULX counterparts ${ }^{6,12}$ (Fig. 2). Note that these WNLs are not WN 5-7 stars, whose spectra are well described with a purehelium models ${ }^{12}$, but LBV-like WN 9-11 stars, which contain up to $70 \%$ hydrogen. Such spectra of high luminosities with prominent He II emission lines have never been observed from any stellarmass black hole X-ray binaries, except for SS 433 and those having WNL donors.

However, neither very hot WNL nor LBV examples exhibit such strong He II $\lambda 4686$ emission lines relative to the hydrogen lines. If the abundance of hydrogen in the ULX donors were 


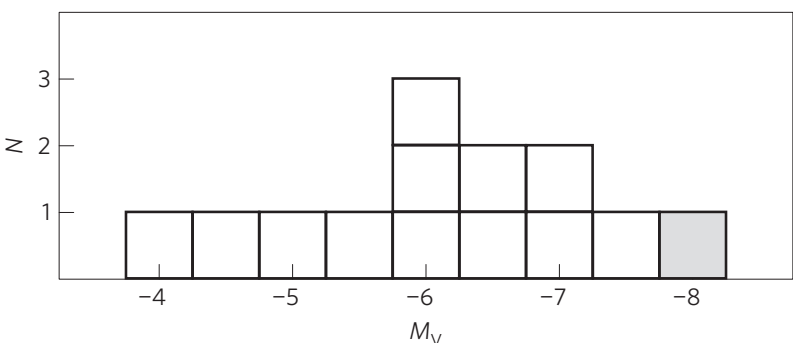

Figure 2 | Absolute magnitudes of all well-studied ULXs. Absolute magnitudes of Holmberg IX X-1, NGC 5204 X-1, NGC 4559 X-7, IC 342 X-1, NGC 5408 X-1, M 81 X-6, M 101 ULX-1, Holmberg II X-1, NGC 6946 ULX-1, NGC 1313 X-1, X-2 (ref. 6) and SS 433 (shadowed) with some updates in the distances in the last four ULXs (ref. 27) and NGC 7793 P13 (ref. 17).

two times smaller from the Solar value, then it could make the $\mathrm{He} / \mathrm{H}$ ratio five times larger, as observed in these ULX spectra (Supplementary Table 1). This contradicts the non-enhancement of the HeI and Pickering He II lines indicating nearly normal abundance of hydrogen. Hence, the wind in the ULXs must be even hotter and more highly ionized than stellar winds in WNL or LBV stars.

All the spectra of the ULXs we obtained are surprisingly similar to one another. The averaged width (FWHM) of the He II line and of $\mathrm{H}_{\alpha}$ are approximately $870 \mathrm{~km} \mathrm{~s}^{-1}$ and $1,000 \mathrm{~km} \mathrm{~s}^{-1}$, respectively. Strikingly, we find that almost all ULX counterparts ever spectroscopically observed exhibit spectra similar to those of WN 9-11 stars, particularly in their broad and strong $\mathrm{He}$ II emission, including NGC 5408 X-1, NGC 1313 X-2, M81 X-6 and M 101 ULX-1 (refs 13-16). In Fig. 3 we plot the $\mathrm{He}$ II and $\mathrm{H}_{0}$ linewidths of our four objects, supplemented with NGC 5408 X-1, which has a simultaneous spectrum including both $\mathrm{He}$ II and $\mathrm{H}_{\alpha}$.

We study the spectra of the ULX counterparts in the He II diagram $^{7}$, where the relation between the linewidth and equivalent width of the He II line is plotted (Supplementary Fig. 2). Here the linewidth represents the terminal velocity of a stellar wind, whereas the equivalent width reflects its photosphere temperature and massloss rate. We also include hottest transition stars from O2If to WN7ha (ref. 9), recorded LBV transitions ${ }^{8}$, and SS 433 (ref. 10). The ULXs and SS 433 occupy a region of the hottest transition stars O2If/WN5-O3.5If/WN7 stars ${ }^{9}$. However, their behaviour in the He II diagram is nothing like stars. They exhibit night-tonight variability by a factor of $2-3$ both in the linewidth and equivalent width. Variability in the radial velocity of the line is also detected, with amplitudes ranging from $100 \mathrm{~km} \mathrm{~s}^{-1}$ in Holmberg IX to $350 \mathrm{~km} \mathrm{~s}^{-1}$ in NGC 5204 (Supplementary Fig. 3).

First, we can exclude the case where these ULXs actually have WNL donors and their stellar winds produce the observed optical spectra. Indeed, the rapid variability of the $\mathrm{He}$ II linewidth is difficult to explain, because the wind terminal velocity in stars is determined by the surface gravity. A more critical problem is that a WNL star means a wind-fed accretion regime, which is not effective ${ }^{16}$. To provide the observed X-ray luminosity, $L_{\mathrm{X}} \sim 10^{40} \mathrm{ers} \mathrm{s}^{-1}$, one needs an unrealistically powerful wind from the donor if the accretor is a stellar-mass black hole. If the primary star were an intermediate mass black hole (IMBH), then the variability of the donor's orbital velocity would be much greater than observed (see Supplementary Section 4 for details). Another obvious argument is the high-quality optical spectrum of NGC 7793 P13 indicating a B9Ia supergiant companion $^{17}$, which itself cannot produce the He II emission line. Therefore, the He II line observed in NGC 7793 P13 must be formed in the second companion, namely, in a photoionized wind from the accretion disk (Supplementary Section 4).

We next consider a possibility that the He II line is formed in the standard accretion disk around a massive black hole. To produce

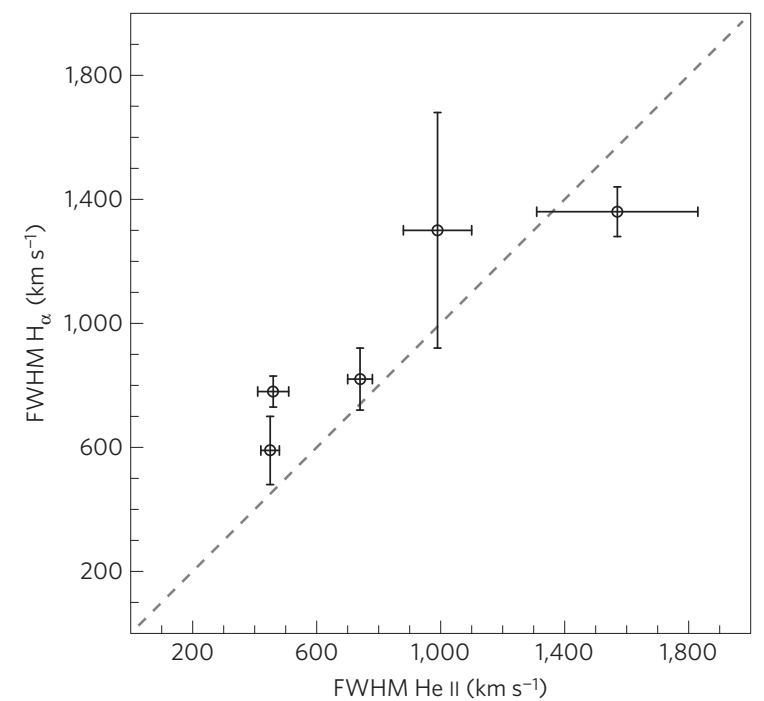

Figure 3 | Emission linewidths. Emission linewidths of $\mathrm{He} \mathrm{II}$ and $\mathrm{H}_{\alpha}$ of Holmberg II X-1, NGC 4559 X-7, NGC 5408 X-1, Holmberg IX X-1 and NGC 5204 X-1, from left to right, all extracted from simultaneous spectra. Systematic errors are taken into account in the error bars of $\mathrm{H}_{\alpha}$ because of the nebula line subtraction. The error on the He II linewidth of NGC $5204 X-1$ is large because of its fainter flux. Note that the averaged He II linewidth of NGC $1313 \mathrm{X}-2$ is $580 \mathrm{~km} \mathrm{~s}^{-1}$ (ref. 14) measured in the spectra without $\mathrm{H}_{\alpha}$.

lines in emission, a strong irradiation of the disk surface by the central source is required. The He II lines trace hotter regions located closer to the black hole than the hydrogen lines, and therefore the He II linewidth is expected to be notably larger than that of $\mathrm{H}_{\alpha}$ in a normal geometry. Indeed, broad $\mathrm{He}$ II and hydrogen emission lines from the self-irradiated disk are observed in Galactic stellar-mass black hole X-ray binaries in outbursts, such as V404 Cyg (ref. 18), GRO J1655-40 (refs 19,20) and GX 339-4 (ref. 21). In all these cases, the He II emission lines are exactly broader than the hydrogen lines. These sources did not reach the supercritical regime; the most reliable determination of the distance towards V404 Cyg based on the astrometric VLBI observations indicates that the luminosity was not super-Eddington during its famous 1989 outburst $^{22}$. We can interpret that their irradiated disks are not blocked by disk winds completely, and hence we observe the $\mathrm{He}$ II emission directly at the disk surface. By contrast, the ULX spectra show that the He II line is narrower than the $\mathrm{H}_{\alpha}$ (Fig. 3) line. It is impossible to explain this fact by irradiated disks unless exotic models are invoked.

We finally examine if the optical spectra of our ULXs can be explained by a supercritical accretion disk (SCAD) with a stellarmass black hole. Indeed, the only known supercritical accretor SS 433 shows a similar optical spectrum to ours, which is produced by the disk wind from the SCAD (ref. 3). In SS 433 the He II line is narrower than $\mathrm{H}_{\alpha}$ (refs 10,23). The same is valid in WNL stars and LBV in their hot state ${ }^{9,24}$. This suggests that the He II and $\mathrm{H}_{\alpha}$ lines are formed in different parts of radiatively accelerated disk winds, where more ionized gas located closer to the source has smaller outflow velocities.

In Supplementary Table 1, we present the mean equivalent widths (relative fluxes) of main emission lines in the ULX spectra with respect to SS 433. Although the line fluxes in all our ULXs are very similar, the indicated ionization degrees are higher than that of SS 433; the hydrogen and He I lines are approximately 12 times weaker than those in SS 433, whereas the two observed He II lines are only two times weaker. These testify less dense but hotter disk winds than that in SS 433. 
Whereas the luminosity is proportional to the mass accretion rate in standard disks, it is expected to have a logarithmic dependence in SCADs (refs 5,25; Supplementary Section 5), because the excess gas is expelled as a disk wind and the accreted gas is advected with the photon trapping, contributing little to the photon luminosity. Instead, the SCAD luminosity has the same order as the Eddington luminosity, $L_{\text {Edd }} \approx 1.5 \times 10^{39} \mathrm{~m}_{10} \mathrm{erg} \mathrm{s}^{-1}$, where $m_{10}$ is the black hole mass in units of 10 solar masses. The mass accretion rate can provide a factor of several in the logarithmic term. Moreover, the funnel in the SCAD wind will collimate the X-ray radiation to an observer also with a factor of several when observed with an inclination angle smaller than 40-50 degrees ${ }^{26}$. Thus, the apparent $\mathrm{X}$-ray luminosity of an ULX with a stellar-mass black hole may well be up to $\sim 10^{41} \mathrm{erg} \mathrm{s}^{-1}$. The SCADs are able to account for the huge $\mathrm{X}$-ray luminosities of the ULXs.

The ultraviolet and optical luminosity may depend strongly on the original mass accretion rate $\dot{M}_{0}$, because these budgets are mainly produced by reprocessing of the strong irradiation from the SCAD's wind (the excess gas). Using simple relations for the SCADs, we find that the optical luminosity of the wind $L_{\mathrm{V}} \propto \dot{M}_{0}^{9 / 4}$ and the wind temperature $T \propto \dot{M}_{0}^{-3 / 4}$ (see Supplementary Section 5 for a more detailed discussion). Accordingly, we find that the mass accretion rates in the ULXs listed in Fig. 2 may be by a factor of 1.5-6 smaller and their wind temperatures a factor of 1.4-4 higher than those in SS 433. SCAD models can explain both the relative dimness of the ULXs in the optical band and higher ionization states of their disk winds than those of SS 433. Thus, we can interpret that SS 433 is intrinsically the same as ULXs, but an extreme case with a particularly high mass accretion rate, which could explain the presence of its persistent jets.

Received 14 November 2014; accepted 30 April 2015; published online 1 June 2015

\section{References}

1. Makishima, K. et al. The nature of ultraluminous compact X-ray sources in nearby spiral galaxies. Astrophys. J. 535, 632-643 (2000).

2. Feng, H. \& Soria, R. Ultraluminous X-ray sources in the Chandra and XMM-Newton era. New Astron. Rev. 55, 166-183 (2011).

3. Fabrika, S. The jets and supercritical accretion disc in SS 433. Astrophys. Space Phys. Rev. 12, 1-152 (2004).

4. Madau, P. \& Rees, M. J. Massive black holes as population III remnants. Astrophys. J. 551, L27-L30 (2001).

5. Poutanen, J., Lipunova, G., Fabrika, S., Butkevich, A. G. \& Abolmasov, P. Supercritically accreting stellar mass black holes as ultraluminous X-ray sources. Mon. Not. R. Astron. Soc. 377, 1187-1194 (2007).

6. Tao, L., Feng, H., Grisé, F. \& Kaaret, P. Compact optical counterparts of ultraluminous X-ray sources. Astrophys. J. 737, 81 (2011).

7. Crowther, P. A. \& Smith, L. J. Fundamental parameters of Wolf-Rayet stars. VI. Large Magellanic Cloud WNL stars. Astron. Astrophys. 320, 500-524 (1997).

8. Sholukhova, O. N., Fabrika, S. N., Zharova, A. V., Valeev, A. F. \& Goranskij, V. P. Spectral variability of LBV star V 532 (Romano's star). Astrophys. Bull. 66, 123-143 (2011).

9. Crowther, P. A. \& Walborn, N. R. Spectral classification of O2-3.5 If*/WN5-7 stars. Mon. Not. R. Astron. Soc. 416, 1311-1323 (2011).

10. Kubota, K. et al. Subaru and Gemini observations of SS 433: New constraint on the mass of the compact object. Astrophys. J. 709, 1374-1386 (2010).

11. Fuchs, Y., Koch Miramond, L. \& Ábrahám, P. SS 433: A phenomenon imitating a Wolf-Rayet star. Astron. Astrophys. 445, 1041-1052 (2006).
12. Hamann, W-R. \& Koesterke, L. The nitrogen spectra of Wolf-Rayet stars. A grid of models and its application to the Galactic WN sample. Astron. Astrophys. 333, 251-263 (1998).

13. Cseh, D. et al. Towards a dynamical mass of the ultraluminous X-ray source NGC 5408 X-1. Mon. Not. R. Astron. Soc. 435, 2896-2902 (2013).

14. Roberts, T. P. et al. (No) dynamical constraints on the mass of the black hole in two ULXs. Astron. Nachr. 332, 398-401 (2011).

15. Bregman, J. N., Felberg, J. N., Seitzer, P. J., Liu, J. \& Kümmel, M. Ultraviolet Spectra of ULX Systems. Preprint at http://arXiv.org/abs/1205.0424 (2012).

16. Liu, J., Bregman, J. N., Bai, Y., Justham, S. \& Crowther, P. Puzzling accretion onto a black hole in the ultraluminous X-ray source M101 ULX-1. Nature 503, 500-503 (2013)

17. Motch, C., Pakull, M. W., Soria, R., Grisé, F. \& Pietrzyski, G. A mass of less than 15 solar masses for the black hole in an ultraluminous X-ray source. Nature 514, 198-201 (2014).

18. Charles, P. A. et al. The bright X ray transient GS2023+338 (=V404 Cyg) in optical outburst and decline. ESASP 296, 103-107 (1989).

19. Hunstead, R. W., Wu, K. \& Campbell-Wilson, D. New radio and optical data for GRO J1655-40. ASPC 121, 63-67 (1997).

20. Soria, R., Wickramasinghe, D. T., Hunstead, R. W. \& Wu, K. Measuring the motion of the black hole in GRO J1655-40. Astrophys. J. 495, L95-L98 (1998).

21. Soria, R., Wu, K. \& Johnston, H. M. Optical spectroscopy of GX 339-4 during the high-soft and low-hard states-I. Mon. Not. R. Astron. Soc. 310, 71-77 (1999).

22. Miller-Jones, J. C. A. et al. The first accurate parallax distance to a black hole. Astrophys. J. 706, L230-L234 (2009).

23. Grandi, S. A. \& Stone, R. P. S. SS 433-How the moving lines move. Publ. Astron. Soc. Pacif. 94, 80-86 (1982).

24. Walborn, N. R. \& Fitzpatrick, E. L. The OB Zoo: A digital atlas of peculiar spectra. Publ. Astron. Soc. Pacif. 112, 50-64 (2000).

25. Shakura, N. I. \& Sunyaev, R. A. Black holes in binary systems. Observational appearance. Astron. Astrophys. 24, 337-355 (1973).

26. Ohsuga, K. \& Mineshige, S. Global structure of three distinct accretion flows and outflows around black holes from two-dimensional radiation-magnetohydrodynamic simulations. Astrophys. J. 736, 2 (2011).

27. Vinokurov, A., Fabrika, S. \& Atapin, K. Ultra-luminous X-ray sources as supercritical accretion discs: Spectral energy distributions. Astrophys. Bull. 68, 139-153 (2013)

\section{Acknowledgements}

The authors are grateful to V. Shimansky and D. Nogami for helpful comments, and T. Hattori for his support in our observations. The research was supported by the Russian RFBR grant 13-02-00885, the Program for Leading Scientific Schools of Russia N 2043.2014.2, the Russian Scientific Foundation (grant N 14-50-00043) and the JSPS KAKENHI Grant number 26400228. S.F. acknowledges the support of the Russian Government Program of Competitive Growth of Kazan Federal University. Based on data collected at Subaru Telescope, which is operated by the National Astronomical Observatory of Japan and on data obtained from the ESO Science Archive Facility (NGC 5408 X-1, ID 385.D-0782).

\section{Author contributions}

S.F. and Y.U. proposed the observations and wrote the paper with comments from all authors. S.F., Y.U. and M.S. carried out the observations. A.V. and O.S. prepared the observational details and reduced the data. S.F., Y.U. and A.V. discussed the results and overall science case with contributions from the remaining authors.

\section{Additional information}

Supplementary information is available in the online version of the paper. Reprints and permissions information is available online at www.nature.com/reprints. Correspondence and requests for materials should be addressed to S.F.

\section{Competing financial interests}

The authors declare no competing financial interests. 